\title{
Análisis Estadístico en Aplicación de Soldadura GTAW Usando Diseño de Experimentos Factorial Completo
}

Tania Elizabeth Sandoval Valencia ${ }^{1}$ (D) , Luis Eduardo Ugalde Caballero ${ }^{1}$, Adriana del Carmen Téllez Anguiano ${ }^{1}$, Héctor Javier Vergara Hernández ${ }^{1}$, Dante Ruiz Robles ${ }^{1}$

${ }^{1}$ Instituto Tecnológico de Morelia, Tecnológico Nacional de México, Morelia, Michoacán, México.

Como citá: Sandoval Valencia TE, Ugalde Caballero LE, Téllez Anguiano AC, Vergara Hernández HJ, Ruiz Robles D. Análisis estadístico en aplicación de soldadura GTAW usando diseño de experimentos factorial completo. Soldagem \& Inspeção. 2020;25:e2516. https://doi.org/10.1590/0104-9224/SI25.16

Resumen: La determinación de los parámetros eléctricos y funcionales para aplicación de soldadura requiere realizar pruebas preliminares tanto en la industria como en la investigación. Sin embargo, este proceso resulta ser muy costoso, lento $\mathrm{y}$, para objeto de investigación, de baja confiabilidad, por tal motivo, el desarrollo de una metodología de experimentación que permita obtener adecuadamente dichos parámetros es muy importante. El presente trabajo muestra el análisis estadístico para la aplicación de soldadura de arco metálico con electrodo de tungsteno y gas inerte (GTAW) utilizada para determinar la velocidad de avance de la antorcha y la corriente de soldadura óptimos para lograr soldadura libre de defectos. Con los resultados obtenidos en el diagrama causa-efecto (Ishikawa) se permite la interpretación efectiva de la causa raíz de los defectos, reduciendo el número de experimentos, posteriormente el diagrama de árbol da pauta para definir la matriz experimental. La validación de la experimentación se hace con el diseño de experimento factorial completo, la efectividad proporcionada por el cálculo del coeficiente de determinación, también conocido como nivel de correlación, de $94,24 \%$ en penetración del cordón y $85,03 \%$ en ancho del cordón. La gráfica de residuales y valores predichos valida la distribución del error en las probetas.

Palabras clave: GTAW; Acero al carbono; Diseño de experimentos.

\section{Statistical Analysis in GTAW Welding Application Using Full Factorial Design Experiment}

\begin{abstract}
Electrical and functional parameters determination for welding application requires preliminary tests in industry and research fields. However, this process turns out to be very expensive, slow and, for research purposes, with low reliability. Thus, the development of a statistical analysis that allows obtaining these parameters properly is very important. The present work shows and experimental methodology to apply the metal arc welding with tungsten electrode and inert gas (GTAW), used to determine the optimum torch speed and current level to achieve defect-free welding. With the results obtained in the cause-effect diagram (Ishikawa), the effective interpretation of the root cause of the defects is allowed, reducing the number of experiments, then the tree diagram gives guidelines to define the experimental matrix. The validation of the experimentation is performed by designing a complete factorial experiment, the effectiveness provided by coefficient of determination estimation or correlation level known is $94.24 \%$ in cord penetration and $85.03 \%$ in cord width. Residuals and predicted values graph are used to validate the specimens error distribution.
\end{abstract}

Key-words: GTAW; Carbon steel; Experiment design.

\section{Introducción}

La soldadura de arco eléctrico con electrodo de tungsteno y gas Inerte (Gas Tungsten Arc Welding, GTAW) es un proceso que utiliza un arco entre un electrodo de tungsteno (no consumible) y el charco de soldadura, el cual se ha vuelto una herramienta indispensable en muchas industrias en virtud de la alta calidad de las soldaduras que produce y del bajo costo del equipo [1-3]; la validez del proceso radica en obtener las características metalúrgicas adecuadas según la aplicación de la pieza soldada. La eficiencia de la experimentación en soldadura se puede validar usando diseño de experimentos; un experimento consiste en un conjunto de actividades, ejecutadas bajo un determinado plan de actuación, que sirven para obtener y analizar datos de forma eficiente y económica [4,5], la experimentación como proceso se puede desarrollar formulando la hipótesis, el diseño de experimentos, el desarrollo experimental, el análisis estadístico de los datos y finalmente las conclusiones [6].

Recibido: 05 Dic., 2019. Aprobado: 15 Abr., 2020

E-mail: D10020080@itmorelia.edu.mx (TESV)

Este es un artículo publicado en acceso (Open Access) abierto bajo la licencia Creative Commons Attribution Non-Commercial, que permite su uso, distribución y reproducción en cualquier medio, sin restricciones siempre que sin fines comerciales y que el trabajo original sea debidamente citado. 
Los parámetros que se deben regular en la aplicación de soldadura son voltaje, corriente, tipo de electrodo, longitud del arco, velocidad de avance de la antorcha, entre otras. Las primeras dos tienen un efecto significativo en la forma del cordón y algunos defectos. La máquina de soldar puede usar un transformador, un inversor de estado sólido o un motor generador para reducir el voltaje de 120, 240 o $480 \mathrm{~V}$ de la línea terminal o de circuito abierto especificado, así como proporcionar corriente elevada, generalmente de orden de 30 a 1500 A, apropiados para la soldadura de arco [3]. Un transformador se define como una máquina estática que tiene la misión de transmitir, mediante un campo electromagnético alterno, la energía eléctrica de un sistema, con determinada tensión, a otros sistemas de tensión deseada [7-10]. Por tal motivo es importante tomar en consideración el ruido que proporciona la máquina de soldar y las condiciones externas, para validar que tan confiables son los resultados experimentales.

Algunos investigadores usan la estadística como apoyo para el análisis de los experimentos; en el 2014 Cruz et al. [11] obtienen los criterios de calidad para mejor el proceso GTAW, el número de experimentos se reduce mediante el uso del análisis de modo y efecto "AMEF" y los resultados se analizan con un diseño central compuesto, obteniendo un nivel de correlación superior al $80 \%$. Costa et al. [12] utilizan la metodología de superficie de respuesta para el modelado de funciones objetivo (variables de respuesta con variables de entrada) y la optimización se realiza con algoritmos genéticos y la gráfica de frente de Pareto, siendo posible establecer modelos matemáticos empíricos satisfactorios para las variables de respuesta. Posteriormente, los autores en [13] realizan un estudio experimental con 15 operadores en el cual se estudia el sesgo de la eficiencia del arco, dando como resultado que el sesgo primordialmente es provocado por la caída del voltaje debido al mal estado de los cables de soldadura, siendo insignificante el factor de la manipulación del operador; estos resultados se evalúan estadísticamente.

Recientes investigaciones utilizan la estadística para obtener parámetros óptimos y mejorar la calidad de los productos o experimentos realizados. Park et al. [14] utilizan el método Taguchi para el diseño de una cosechadora que garantice un voltaje de salida suficiente alto y robusto en un amplio rango de frecuencia producido por vibraciones mecánicas externas, confirmando que la longitud del sustrato tiene mayor influencia en la respuesta del recolector, con 18 experimentos se obtiene la combinación óptima resultando una reducción de aproximadamente $9,86 \%$ para el voltaje de salida; el valor de desviación se reduce en aproximadamente 18,14\%. Investigadores utilizan el método Taguchi [15-20] para optimizar soldadura de puntos por resistencia (RSW) de un acero DP-290; obteniendo que una menor intensidad de corriente y presión moderada junto con mayores tiempos de soldeo producen una mejor resistencia al desgaste.

La presente investigación se delimita al uso del proceso GTAW para la obtención de raíz de soldadura con penetración completa y con buena fusión en el ancho del cordón, en el cual se mantienen fijos la mayoría de los parámetros para mostrar que realizando de forma correcta la planeación de los experimentos se pueden obtener resultados confiables sin sesgo (análisis estadístico) con pocas pruebas. Es importante resaltar que con solo nueve experimentos en un acero al carbono se obtuvieron los parámetros óptimos para raíz de soldadura con penetración completa y ancho de cordón adecuado, se observa que los errores de experimentación no fueron concentrados en una sola probeta comprobando con ello la confiabilidad de los resultados experimentales.

En el presente trabajo, los factores controlables y las variables independientes durante la aplicación del proceso de soldadura se delimitan con el uso del diagrama causa-efecto (Ishikawa), el cual es una herramienta que identifica cada defecto del cordón con el parámetro eléctrico o el factor externo que lo ocasiona.

\section{Metodología}

Existen filosofías que proponen etapas para la calidad del producto fabricado como es el caso de Taguchi que se basa en tres conceptos fundamentales. En primer lugar, considera que el diseño en la fase inicial del producto es fundamental para lograr la calidad en el resultado final. Por otra parte, sostiene que la calidad de los productos mejora cuando se minimiza la variabilidad en torno al valor nominal u objetivo. Por último, entiende a los costos de la no calidad como función de la variabilidad en los procesos [21].

Tomando en consideración la filosofía de calidad de Taguchi se desarrolló la metodología utilizada para la planeación y análisis de las variables de entrada (parámetros controlables y controlables) y salida (efecto de correlación) a evaluar para la presente investigación. Como se muestra a continuación:

1. Diseño del diagrama causa-efecto, con la cual se reducen el número de experimentos;

2. Realización del diagrama de árbol como base del diseño de la matriz experimental, permitiendo generar el control de las variables controlables;

3. Generación de la matriz experimental contemplando dos factores con tres niveles, estableciendo un diseño de experimentos factorial completo $3^{\wedge} \mathrm{K}$. Es importante resaltar que en esta esta etapa se determina las variables de salida, también conocidas como de interés;

4. Realización de experimentos;

5. Análisis estadístico; 
6. Aplicación de soldadura con los parámetros analizados estadísticamente, con lo cual se comprueba la confiabilidad del análisis estadístico sin réplicas es confiable, al obtener las características deseadas en el cordón de soldadura.

\subsection{Diagrama causa y efecto (Ishikawa)}

Se realiza como primera etapa el diagrama causa-efecto de Ishikawa que identifica el defecto del cordón con el parámetro que lo provoca (Figura 1), para establecer los factores o causa raíz que ocasionan los defectos que se detectan posteriormente a la aplicación de soldadura, permitiendo determinar los factores controlables y variables independientes de la matriz experimental, lo cual es importante para la reducción del número de experimentos.

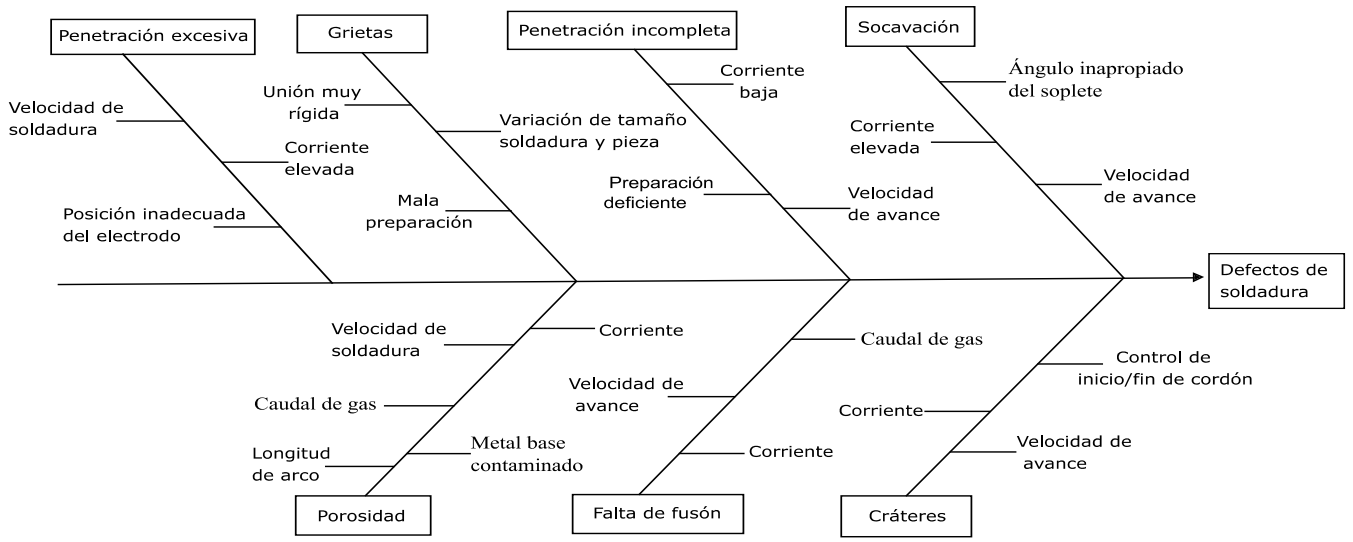

Figura 1. Diagrama Causa-Efecto (Ishikawa) de defectos de soldadura [22-24].

Al analizar el diagrama causa-efecto Ishikawa las causas principales que ocasionan los diferentes defectos en el proceso de soldadura de arco metálico con electrodo de tungsteno y gas inerte (GTAW) semiautomatizado son los siguientes:

1. El defecto de soldadura porosidad (bolsas de gas que se quedan retenidas en la soldadura o abiertas a la superficie), ocurre comúnmente por un bajo caudal de gas protector o el uso de gas protector contaminado o incorrecto, en otros casos la corriente de soldadura y velocidad de avance excesivo o metal base contaminado;

2. La penetración excesiva (exceso material de soldadura respecto a la cantidad requerida para rellenar la junta), este problema puede ser causado por el uso de corriente de soldadura muy alta, también de un avance demasiado lento, en ambas situaciones, se consume mucho más metal de aportación de lo que se requeriría normalmente, también la posición inadecuada del electrodo puede provocar el defecto;

3. Penetración incompleta, se produce cuando la corriente de soldadura está regulada demasiado baja, el avance demasiado rápido también puede producir penetración insuficiente, en ambos casos puede ocurrir la fusión incompleta a lo largo del contorno de la soldadura. La preparación inadecuada de la probeta puede provocar la aparición del mismo;

4. La socavación (ranura en el material adyacente a la soldadura) es provocada por una velocidad de avance alta o corriente de soldadura inadecuada, ya que no permite que una cantidad suficiente de metal de aporte se funda y se deposite sobre la zona soldada hasta el nivel de la superficie del metal base, también el ángulo de trabajo inapropiado produce soldaduras de filete de catetos dispares;

5. El defecto de falta de fusión sucede cuando el arco no consigue fundir el material base, ocasionado por corriente de soldadura y velocidad de soldadura inadecuada, también el ángulo incorrecto de la antorcha y una selección inadecuada del gas de protección.

Como factores controlables se opta por mantener fijos la composición del aporte, la posición de la antorcha, el tamaño de las probetas, la dimensión y el acabado de la punta de electrodo de tungsteno no consumible, así como la limpieza de la probeta a soldar. Las variables independientes son la velocidad de avance de la antorcha y la corriente de soldadura de la máquina de soldar.

El diseño de la probeta para aplicación de soldadura también se considera un factor controlable (todas las probetas para

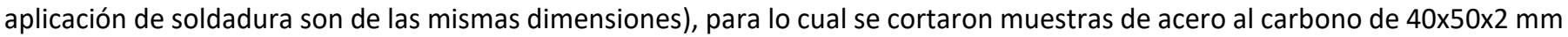
con la cortadora de disco para ser careadas, biseladas y barrenadas posteriormente con la fresadora Great Machine Tools ${ }^{\circledR}$, esto con el fin de obtener un paralelismo entre las caras de las muestras con un ángulo de sesenta grados y con una cara de la raíz de $2 \mathrm{~mm}$, con una separación de raíz de 1,6 mm obteniendo las nueve probetas para aplicación de soldadura, como se muestra en la Figura 2. 


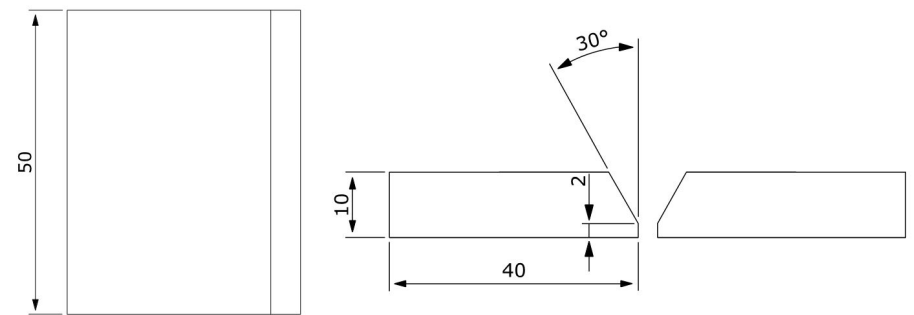

Figura 2. Probeta para aplicación de soldadura.

\subsection{Diagrama de árbol}

Las variables independientes (factores que se varían a nivel experimental para observar el efecto que tienen en las variables de salida) se trabajan en tres niveles de corriente de soldadura y velocidad de avance de la antorcha, para lo cual se presenta un árbol de decisión para determinar las combinaciones de los parámetros en la experimentación, los resultados obtenidos se muestran en la Figura 3. La primera combinación es considerando una velocidad de avance de la antorcha de $0,7 \mathrm{~mm} / \mathrm{s}$ y una corriente de soldadura de $100 \mathrm{~A}$, con incrementos sucesivos de nivel.

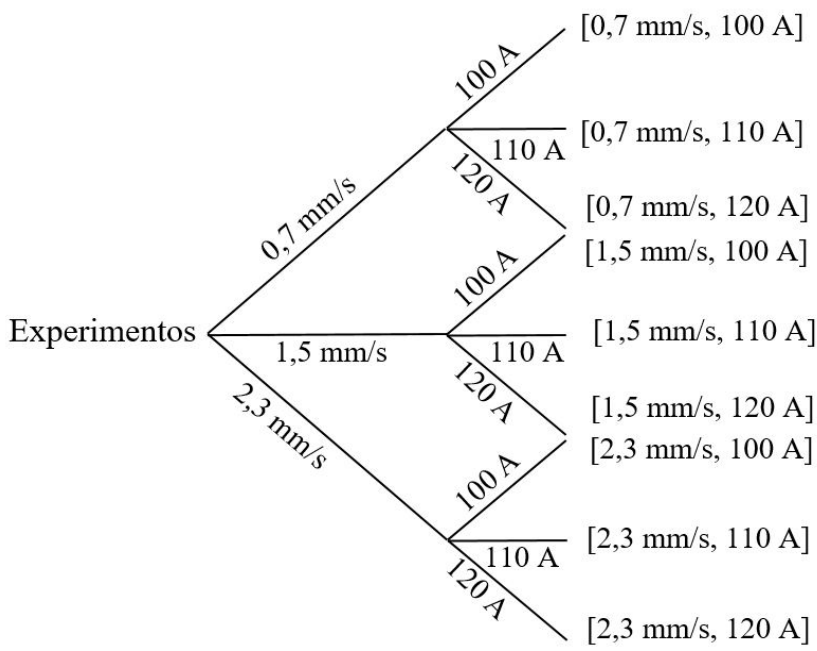

Figura 3. Diagrama de árbol para combinación de niveles experimentales.

Después de establecer las variables independientes se aplica el proceso de soldadura GTAW con una máquina de soldar Arctron 288 HF, INFRA ${ }^{\circledR}$ sobre probetas de acero al carbono A36, con los parámetros que se muestran en la Tabla 1.

Tabla 1. Parámetros del proceso GTAW.

\section{GTAW (TIG)}

Posición de soldadura

Gas de Protección

Acabado del electrodo no consumible

Ángulo de afilado del electrodo

Tipo de electrodo no consumible

Diámetro del electrodo

Parámetros

\section{$1 G$ \\ $\mathrm{Ar}$}

Afilado

$45^{\circ}$

EWTh-2

$1,6 \mathrm{~mm}$

$5,37 \mathrm{~mm}$

\section{Polaridad}

Caudal de Gas

Stick out

Boquilla
Electrodo negativo de corriente continua (CCEN)

$$
\begin{gathered}
11,7987 \mathrm{I} / \mathrm{min} \\
2,53 \mathrm{~mm} \\
13,00 \mathrm{~mm} \\
\text { ER } 70 \mathrm{~S}-3
\end{gathered}
$$

$2,4 \mathrm{~mm} \times 914 \mathrm{~mm}$ 
Es importante resaltar que la velocidad de avance de la antorcha, la separación entre la superficie de trabajo y la punta del electrodo de tungsteno se mantuvieron constantes, ya que se utilizó un tractor de oxicorte CG100 como se muestra en la Figura 4.

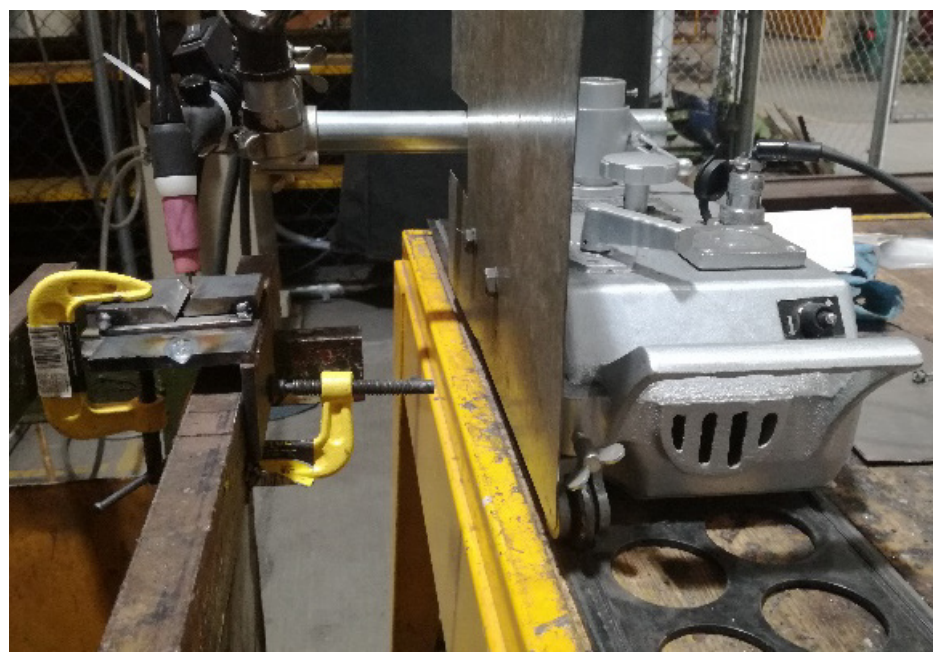

Figura 4. Tractor de oxicorte.

\subsection{Diseño de experimentos factorial completo}

El diseño de experimentos factorial completo $3^{\wedge} \mathrm{K}$ permite determinar la efectividad de los experimentos, así como obtener los parámetros óptimos para una raíz de soldadura GTAW libre de defectos. Las salidas medidas son la penetración y el ancho del cordón se muestra en la Tabla 2. Es importante resaltar que la combinación de los dos factores y tres niveles fueron establecidos de forma estándar.

Finalmente, los factores de entrada y salida fueron analizados con el diseño de experimentos factorial completo usando el programa Statistica ${ }^{\circledR}$.

Tabla 2. Variables independientes y respuestas.

\begin{tabular}{cccc}
\hline Velocidad de avance $(\mathbf{m m} / \mathbf{s})$ & Corriente de soldadura (A) & Penetración del cordón $(\mathbf{m m})$ & Ancho del cordón $(\mathbf{m m})$ \\
0,7 & 100 & 3,69 & 5,10 \\
0,7 & 110 & 3,99 & 6,67 \\
0,7 & 120 & 3,79 & 6,15 \\
1,5 & 100 & 3,10 & 4,74 \\
1,5 & 110 & 2,78 & 4,73 \\
1,5 & 120 & 3,43 & 5,57 \\
2,3 & 100 & 2,38 & 4,01 \\
2,3 & 110 & 2,15 & 3,89 \\
2,3 & 120 & 2,32 & 4,72 \\
\hline
\end{tabular}

\section{Resultados y Discusión}

\subsection{Diseño de experimentos factorial completo}

En contrastes de hipótesis y en estadística general, el valor $p$ (conocido como $p$-valor, valor $p$ consignado, o bien directamente en inglés $p$-value) es la probabilidad de obtener un resultado al menos tan extremo como el que realmente se ha obtenido (valor del estadístico calculado), suponiendo que la hipótesis nula es cierta, en términos de probabilidad condicional. Al analizar las salidas de un diseño de experimentos, la estimación de los efectos y porcentaje de confiabilidad son las variables más importantes.

En la Tabla 3 se puede observar que la velocidad de avance de la antorcha es el parámetro que se considera estadísticamente significativo, llamado efecto principal porque se refiere al interés primario en el experimento [25], al ser el valor $p<0,05$ en la variable de salida penetración del cordón. Si se desea cuidar este factor dependiente es importante tener un control riguroso en la velocidad de avance con la que se está trabajando. De igual manera se observa que la efectividad de la experimentación es del $94,24 \%$ de confianza, obtenida por el cálculo del coeficiente de determinación, r-cuadrado. 
Es importante saber que el resultado del R cuadrado oscila entre 0 y 1 , cuanto más cerca de 1 se sitúe su valor, mayor será el ajuste del modelo a la variable que se intenta explicar.

Tabla 3. Estimación del efecto de los factores en la penetración del cordón.

\begin{tabular}{|c|c|c|c|c|c|c|c|c|c|c|}
\hline \multirow[t]{2}{*}{ Factor } & \multicolumn{10}{|c|}{$\begin{array}{c}\text { Estimación de efectos; Var.: Penetración del cordón; R-sqr=0,94244 } \\
2 \text { factores } 3 \text { niveles, } 1 \text { Blocks, } 9 \text { experimentos } \\
\text { DV: Penetración del cordón }\end{array}$} \\
\hline & Effect & Std.Err. & $t(4)$ & $p$ & $\begin{array}{c}-95 . \% \\
\text { Cnf.Limt }\end{array}$ & $\begin{array}{c}+95 . \% \\
\text { Cnf.Limt }\end{array}$ & Coeff. & $\begin{array}{l}\text { Std.Err. } \\
\text { Coeff. }\end{array}$ & $\begin{array}{c}-95 . \% \\
\text { Cnf.Limt }\end{array}$ & $\begin{array}{c}+95 . \% \\
\text { Cnf.Limt }\end{array}$ \\
\hline Mean/Interc. & 3,07000 & 0,078446 & 39,13543 & 0,000003 & 2,85220 & 3,28780 & 3,070000 & 0,078446 & 2,85220 & 3,287800 \\
\hline (1)Velocidad(L) & $\begin{array}{c}- \\
1,54000\end{array}$ & 0,192152 & $-8,01451$ & 0,001315 & $-2,07350$ & $-1,00650$ & $\begin{array}{c}- \\
0,770000\end{array}$ & 0,096076 & $-1,03675$ & $\begin{array}{c}- \\
0,503251\end{array}$ \\
\hline Velocidad(Q) & 0,05000 & 0,166408 & 0,30047 & 0,778791 & $-0,41202$ & 0,51202 & 0,025000 & 0,083204 & $-0,20601$ & 0,256012 \\
\hline (2)Corriente(L) & 0,12333 & 0,192152 & 0,64185 & 0,555902 & $-0,41016$ & 0,65683 & 0,061667 & 0,096076 & $-0,20508$ & 0,328416 \\
\hline Corriente(Q) & $\begin{array}{c}- \\
0,14500\end{array}$ & 0,166408 & $-0,87135$ & 0,432738 & $-0,60702$ & 0,31702 & $\begin{array}{c}- \\
0,072500\end{array}$ & 0,083204 & $-0,30351$ & 0,158512 \\
\hline
\end{tabular}

Nota: Std.Err.: error estándar.

En la Tabla 4 se puede observar que la velocidad de avance nuevamente es el factor estadísticamente significativo en el ancho del cordón del proceso GTAW, siendo el R cuadrado de 85,03\% de efectividad en el control de las variables independientes y controlables al momento de realizar la experimentación, concluyendo que los factores controlables fueron determinados de forma adecuada y por lo tanto no se tiene ruido en la correlación con las salidas medidas.

Tabla 4. Estimación del efecto de los factores en el ancho del cordón.

\begin{tabular}{|c|c|c|c|c|c|c|c|c|c|c|}
\hline \multirow[t]{2}{*}{ Factor } & \multicolumn{10}{|c|}{$\begin{array}{c}\text { Estimación de efectos; Var.: Ancho del cordón; R-sqr }=0,85031 \\
2 \text { factores } 3 \text { niveles, } 1 \text { Blocks, } 9 \text { experimentos } \\
\text { DV: Ancho del cordón }\end{array}$} \\
\hline & Effect & Std.Err. & $t(4)$ & p & $\begin{array}{c}-95 . \% \\
\text { Cnf.Limt }\end{array}$ & $\begin{array}{c}+95 . \% \\
\text { Cnf.Limt }\end{array}$ & Coeff. & $\begin{array}{c}\text { Std.Err. } \\
\text { Coeff. }\end{array}$ & $\begin{array}{c}-95 . \% \\
\text { Cnf.Limt }\end{array}$ & $\begin{array}{c}+95 . \% \\
\text { Cnf.Limt }\end{array}$ \\
\hline Mean/Interc. & 5,06444 & 0,168647 & 30,02977 & 0,000007 & 4,59620 & 5,532685 & 5,064444 & 0,168647 & 4,59620 & 5,532685 \\
\hline (1)Velocidad(L) & $-1,76667$ & 0,413100 & $-4,27660$ & 0,012882 & $-2,91362$ & 0,619716 & $\begin{array}{c}- \\
0,883333\end{array}$ & 0,206550 & $-1,45681$ & $-0,30985$ \\
\hline Velocidad(Q) & $-0,07667$ & 0,357755 & $-0,21430$ & 0,840795 & $-1,06995$ & 0,916621 & $\begin{array}{c}- \\
0,038333\end{array}$ & 0,178878 & $-0,53498$ & 0,458311 \\
\hline (2)Corriente(L) & 0,86333 & 0,413100 & 2,08989 & 0,104844 & $-0,28362$ & 2,010284 & 0,431667 & 0,206550 & $-0,14181$ & 1,005142 \\
\hline Corriente(Q) & 0,04833 & 0,357755 & 0,13510 & 0,899057 & $-0,94495$ & 1,041621 & 0,024167 & 0,178878 & $-0,47248$ & 0,520811 \\
\hline
\end{tabular}

Nota: Std.Err.: error estándar.

La Figura 5a demuestra que los datos de la muestra se ajustan a una distribución normal con un nivel de confianza del $94,24 \%$ según el análisis complementario que es el ANOVA. La gráfica de los residuales que se muestra en la Figura $5 \mathrm{~b}$ denota que los errores presentes en la gráfica de normalidad se encuentran distribuidos en toda la experimentación, manteniendo un rango respecto a la media de $\pm 0,3$. 

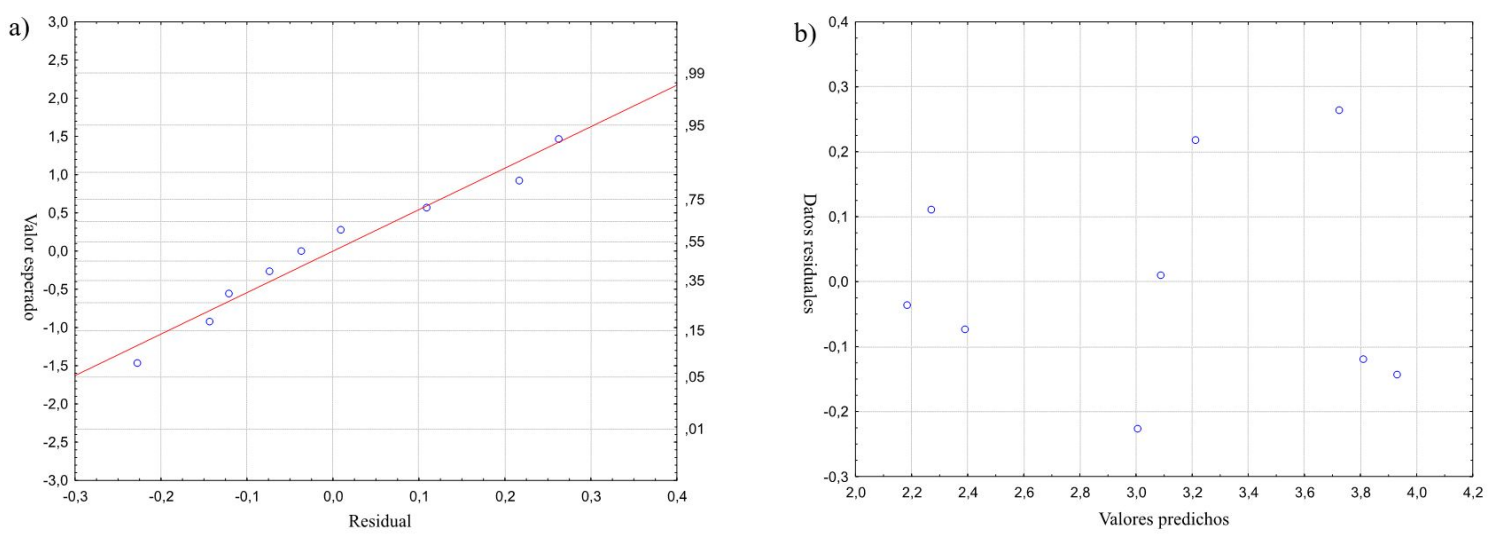

Figura 5. Penetración del cordón: (a) Gráfica de normalidad; (b) Gráfica de residuales.

Con base en el gráfico de normalidad que se muestra en la Figura 6a) se puede observar que los datos presentan una distribución normal. La gráfica de residual en la Figura $6 \mathrm{~b}$ ) muestra que los errores con respecto a la media están distribuidos de manera aleatoria por lo tanto se tiene independencia del error.
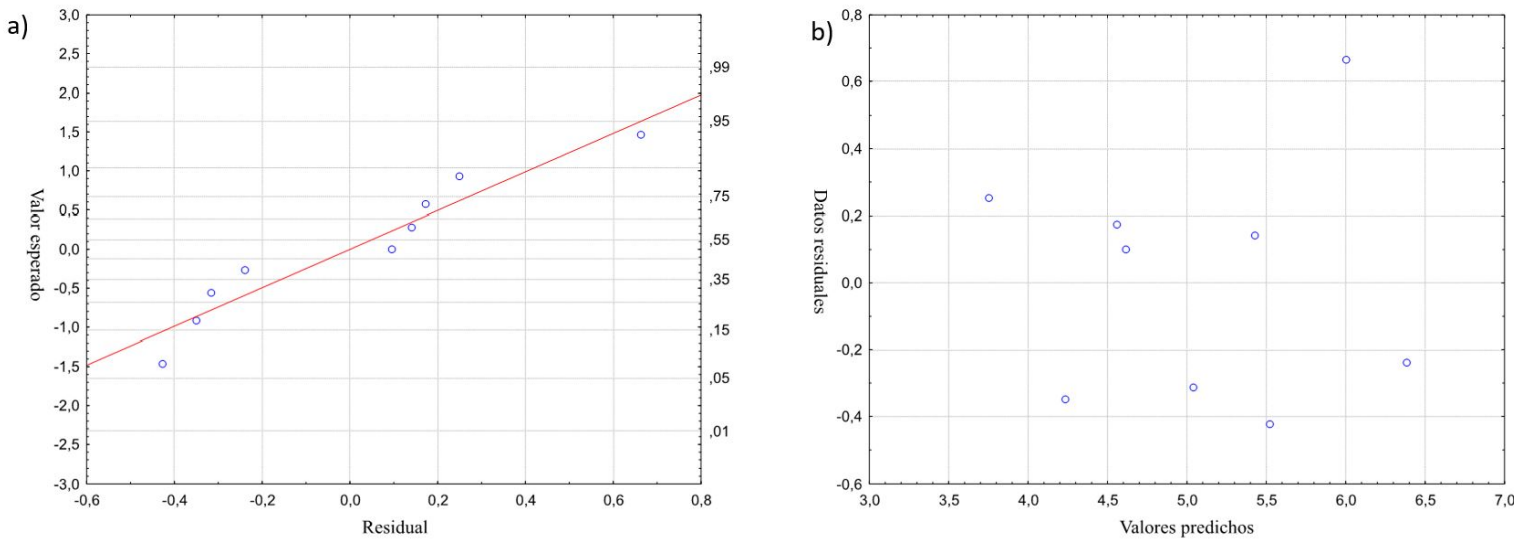

Figura 6. Ancho del cordón: (a) Gráfica de normalidad; (b) Gráfica de residuales.

La estimación de efectos, conocida como ANOVA (Analysis of variance), evalúa la importancia de uno o más factores al comparar las medias de la variable de respuesta en los diferentes niveles. En la Tabla 5 se muestra el análisis de varianza teniendo como salida la penetración del cordón, se puede observar que la velocidad de avance de la antorcha tiene significancia dentro del modelo estadístico estudiado. La corriente de soldadura a nivel experimental no fue considerado estadísticamente significativo, ya que el rango de variación es pequeño entre cada nivel de los factores analizados, es importante resaltar que no se realizan replicas en la experimentación.

Tabla 5. Análisis de varianza de la penetración del cordón.

\begin{tabular}{|c|c|c|c|c|c|}
\hline \multirow[t]{2}{*}{ Factor } & \multicolumn{5}{|c|}{$\begin{array}{l}\text { ANOVA; Var.: Penetración del Cordón }(\mathrm{mm}) \\
9 \text { experimentos; } \mathrm{R} \text {-sqr }=0,94244 \\
\text { Penetración del cordón }(\mathrm{mm})\end{array}$} \\
\hline & SS & df & MS & $\mathbf{F}$ & p \\
\hline (1) Velocidad de avance $(\mathrm{mm} / \mathrm{s})$ (L) & 3,557400 & 1 & 3,5574400 & 64,23232 & 0,001315 \\
\hline Velocidad de avance $(\mathrm{mm} / \mathrm{s})(\mathrm{Q})$ & 0,005000 & 1 & 0,005000 & 0,09028 & 0,778791 \\
\hline (2) Corriente de soldadura (A) (L) & 0,022817 & 1 & 0,022817 & 0,41198 & 0,555902 \\
\hline Corriente de soldadura (A) (Q) & 0,042050 & 1 & 0,042050 & 0,75925 & 0,432738 \\
\hline Error & 0,221533 & 4 & 0,055383 & & \\
\hline Total SS & 3,848800 & 8 & & & \\
\hline
\end{tabular}

Nota: SS; suma de cuadrados, df; grados de libertad de cada suma de cuadrados, MS; cociente entre la suma de cuadrados y sus correspondientes grados de libertad, F; estadístico $\mathrm{F}$, p; valor-p. 
Para el análisis ANOVA del ancho del cordón muestra que también la velocidad de avance de la antorcha es el parámetro que se considera estadísticamente significativo en su forma lineal como se muestra en la Tabla 6.

Tabla 6. Análisis de varianza en el ancho del cordón.

\begin{tabular}{|c|c|c|c|c|c|}
\hline \multirow[t]{2}{*}{ Factor } & \multicolumn{5}{|c|}{$\begin{array}{l}\text { ANOVA; Var.: Ancho del Cordón }(\mathrm{mm}) \\
9 \text { experimentos; } \mathrm{R} \text {-sqr }=0,85031 \\
\text { Ancho del cordón }(\mathrm{mm})\end{array}$} \\
\hline & SS & df & MS & $\mathbf{F}$ & p \\
\hline (1) Velocidad de avance $(\mathrm{mm} / \mathrm{s}$ ) (L) & 4,681667 & 1 & 4,681667 & 18,28935 & 0,012882 \\
\hline Velocidad de avance (mm/s) (Q) & 0,011756 & 1 & 0,011756 & 0,04592 & 0,840795 \\
\hline (2) Corriente de soldadura (A) (L) & 1,118017 & 1 & 1,118017 & 4,36763 & 0,104844 \\
\hline Corriente de soldadura $(\mathrm{A})(\mathrm{Q})$ & 0,004672 & 1 & 0,004672 & 0,01825 & 0,899057 \\
\hline Error & 1,023911 & 4 & 0,255978 & & \\
\hline Total SS & 6,840022 & 8 & & & \\
\hline
\end{tabular}

Nota: SS; suma de cuadrados, df; grados de libertad de cada suma de cuadrados, MS; cociente entre la suma de cuadrados y sus correspondientes grados de libertad, F; estadístico F, p; valor-p.

La gráfica de superficie muestra la relación tridimensional, con variables en los ejes X y Y (corriente de soldadura y velocidad de avance), y la variable de respuesta Z representada la penetración del cordón, en el cual es importante resaltar que la interacción como tal no fue evaluada, ya que se usaron los correspondientes grados de libertad en el error. La Figura 7 muestra el efecto que tiene la velocidad de avance y la corriente de soldadura sobre la penetración del cordón en el proceso GTAW de un acero al carbono, con lo cual se puede deducir que:

1. Para tener una penetración de cordón entre $3,4 \mathrm{~mm}$ a $3,8 \mathrm{~mm}$ es necesario disminuir la velocidad de avance entre 0,7 y $1,2 \mathrm{~mm} / \mathrm{s}$ con una intensidad de corriente que oscila entre $100 \mathrm{~A}$ y $120 \mathrm{~A}$;

2. Para que la penetración sea de aproximadamente de $3,2 \mathrm{~mm}$ y $3,0 \mathrm{~mm}$ se requiere una velocidad de avance entre 1,4 y $1,6 \mathrm{~mm} / \mathrm{s}$ con intensidad de corriente entre los rangos establecidos en la Tabla 2;

3. Si se requiere que la penetración del cordón sea menor a 2,8 $\mathrm{mm}$ se debe aumentar la velocidad de avance a $1,8 \mathrm{~mm} / \mathrm{s}$ o más, con intensidad de corriente entre el rango de $100 \mathrm{~A}$ y $120 \mathrm{~A}$.

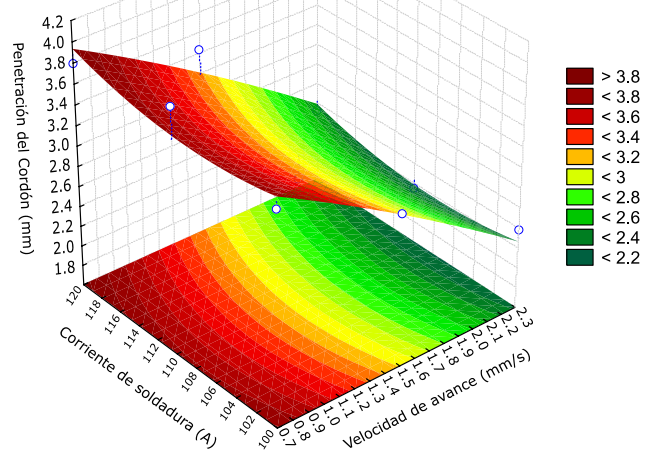

Figura 7. Gráfica de superficie del efecto de la velocidad de avance y corriente de soldadura en la penetración del cordón.

La gráfica de superficies que se presenta en la Figura 8 permite determinar las siguientes características:

1. Para tener un ancho de cordón mayor a $6,0 \mathrm{~mm}$ se requiere una velocidad de avance de $0,7 \mathrm{~mm} / \mathrm{s}$ e intensidad de corriente entre 100 A y $120 \mathrm{~A}$;

2. Si se desea tener un ancho de cordón entre los $4,8 \mathrm{~mm}$ y $5,3 \mathrm{~mm}$ es de gran importancia controlar la velocidad de avance de entre $0,9 \mathrm{~mm} / \mathrm{s}$ a $1,7 \mathrm{~mm} / \mathrm{s}$ la intensidad de corriente puede variar en el rango que se estuvo trabajando en la presente investigación;

3. Si se requiere un ancho de cordón de menor a $4,3 \mathrm{~mm}$ se debe utilizar una velocidad de avance mayor o igual que $1,8 \mathrm{~mm} / \mathrm{s}$. 


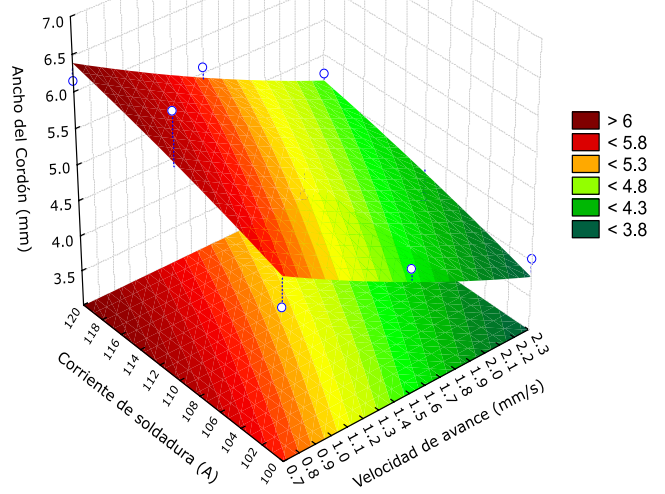

Figura 8. Gráfica de superficie del efecto de la velocidad de avance y corriente de soldadura en el ancho del cordón.

Los parámetros óptimos de soldadura GTAW en el acero A36 para obtener ancho y penetración del cordón de 6,5 mm son corriente de soldadura de 120 A y velocidad de avance del tractor de $0,7 \mathrm{~mm} / \mathrm{s}$. Los resultados se muestran en la Figura 9.
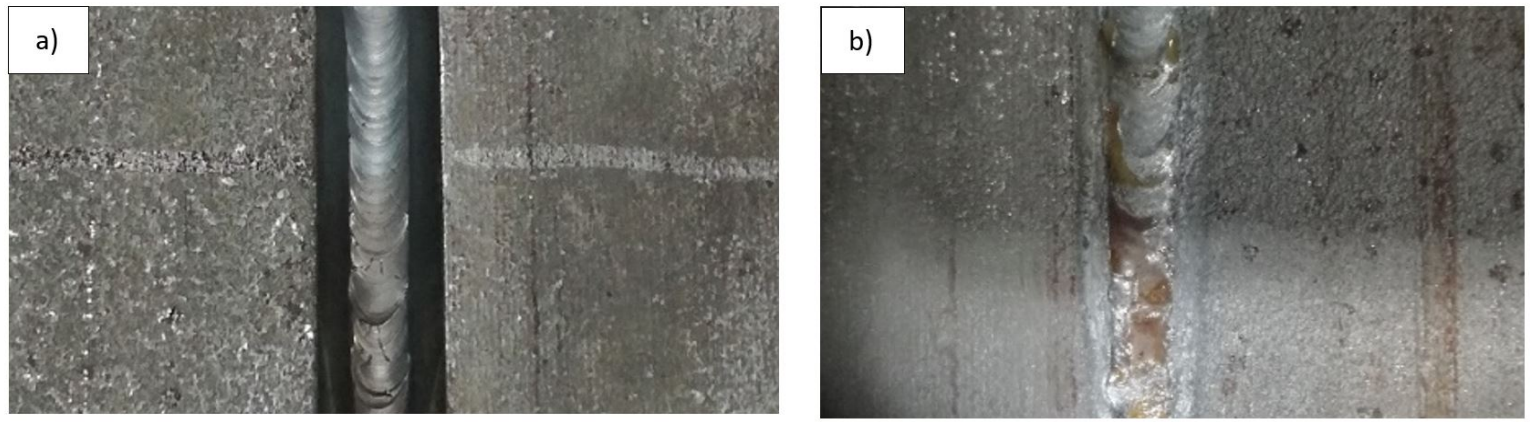

Figura 9. Proceso GTAW (pasada de raíz): (a) Parte superior; (b) Parte inferior.

La soldadura de pasada de raíz tiene penetración completa y un cordón homogéneo sin defectos en la superficie con lo cual se concluye que los parámetros utilizados son adecuados para el acero al carbono.

\section{Conclusiones}

El diagrama causa-efecto conocido como Ishikawa es de gran ayuda a disminuir el número de experimentos de soldadura, optimizando el tiempo y costo pruebas experimentales.

El análisis de penetración y ancho del cordón, muestra que la velocidad de avance de la antorcha tiene un efecto significativo en la penetración y ancho del cordón.

El control de los factores que tienen efecto sobre la penetración y ancho del cordón permite obtener un ajuste del modelo estadístico de $94,24 \%$ y $85,03 \%$ de efectividad y control en la experimentación.

El análisis estadístico puede ser utilizado en soldadura de arco eléctrico con electrodo de tungsteno y gas inerte. Los factores pueden variarse y ajustarse a los niveles deseados para evaluar el comportamiento de la salida medida.

El uso del diseño de experimento factorial completa es una herramienta que se aplica para llevar a cabo la evaluación de los parámetros de entrada respecto a la salida, mostrando una respuesta adecuada, sin embargo, hay diseños de experimentos más eficientes que los $3^{\wedge} \mathrm{k}$ que se pueden aplicar.

El análisis de los resultados experimentales mediante el diseño de experimentos factoriales completos es una gran herramienta para analizar el proceso de soldadura GTAW y obtener resultados confiables, sin embargo, se recomienda realizar replicas en la experimentación para obtener el error experimental.

\section{Agradecimientos}

Se agradece al Tecnológico Nacional de México/Instituto Tecnológico de Morelia y al programa de doctorado (Doctorado en Ciencias en Ingeniería Eléctrica y Doctorado en Ciencias en Ingeniería) por el apoyo brindado para la realización de la presente investigación. También al Consejo Nacional de Ciencia y Tecnología CONACYT. 


\section{Referencias}

[1] Salkin JT, Beedon KW, Henon BK, Jelonek KR, O’Donnell DB. Gas tungsten arc welding. In: American Welding Society. AWS handbook. 2nd ed. Miami: AWS; 2004.

[2] West Arco. Manual de soldadura. Bogotá; 2015.

[3] American Welding Society. Welding handbook: welding processes. 8th ed. Miami: AWS; 1996.

[4] Keppel G. Design and analysis: a researcher's handbook. 3rd ed. New York: Englewood Clifts; 1991.

[5] Gata AM, Siannah DM. Diseño de experimentos aplicado a investigaciones agrícolas relacionadas con el campo electromagnético. Rev Av Científica. 2016;19(1):1-11.

[6] Ruiz de Maya S, López IL. Metodología del diseño experimental. In: Sarabia Sanchez FJ, editor. Métodos de investigación social y de la empresa. Madrid: Ediciones Pirámide; 2015. p. 485-502.

[7] Ruiz-Robles D, Ortíz-Marín J, Venegas-Rebollar V, Moreno-Goytia E, Granados-Lieberman D, Rodríguez-Rodriguez J. Nanocrystalline and silicon steel medium-frequency transformers applied to DC-DC converters: analysis and experimental comparison. Energies. 2019;12(11):2062. http://dx.doi.org/10.3390/en12112062.

[8] Oliva ER. Transformadores de potencia, de medida y de protección. 7. ed. Barcelona: Marcombo; 1994.

[9] Ruiz-Robles D, Venegas-Rebollar V, Anaya-Ruiz A, Moreno-Goytia EL, Rodríguez-Rodríguez JR. Design and prototyping mediumfrequency. Energies. 2018;11(8):2081.

[10] Avelino Pérez P. Transformadores de distribución: teoría, cálculo, construcción y pruebas. 2. ed. Barcelona: Reverté; 2001.

[11] Cruz C, Hiyane G, Mosquera-Artamonov JD, Salgado L JM. Optimización del proceso de soldadura GTAW en placas de Ti6Al4V. Soldagem e Inspeção. 2014;19(1):2-9. http://dx.doi.org/10.1590/S0104-92242014000100002.

[12] Costa PS, Reyes-Valdés FA, Saldaña-Garcés R, González-González DS, Delgado-Albavera ER. Optimización de los parámetros de soldadura por arco sumergido en acero HSLA: una aplicación para manufactura de tuberías de conducción de hidrocarburos optimization of welding parameters in HSLA steel using SAW process. Soldagem e Inspeção. 2015;20(4):456-466. http://dx.doi.org/10.1590/01049224/SI2004.12.

[13] Sikström F. Operator bias in the estimation of arc efficiency in gas tungsten arc welding. Soldagem e Inspeção. 2015;20(1):128-133. http://dx.doi.org/10.1590/0104-9224/SI2001.13.

[14] Park S, Kim H, Kim J, Lee TH, Cho S. Taguchi design of PZT-based piezoelectric cantilever beam with maximum and robust voltage for wide frequency range. Electron. Mater. 2019;48(11):6881-6889. http://dx.doi.org/10.1007/s11664-019-06994-1.

[15] Thakur AG, Rao T, Mukhedkar MS, Nandedkar VM. Application of Taguchi method for resistance spot welding of galvanized steel. Journal of Engineering and Applied Sciences. 2010;5(11):22-26.

[16] Kumar D, Kumar V. Parametric optimization through Taguchi approach for tensile stress in FSW of AA6101-T6. Revista Internacional de Pesquisa e Tecnologia em Engenharia. 2018;6(16):1-4.

[17] Singh S, Singh K, Singh I, Shivesh C. An experimental analysis and optimization of process parameters on friction stir welding of dissimilar AA6061-T6 and AA6951-T6 using taguchi technique. International Research Journal of Engineering and Technology. 2017;4(7):33293335.

[18] Reyes-Calderón F, Vences-Hernández R, Salazar-Torres JA, Vergara-Hernández HJ, Aguilera-Navarrete I, Pérez-González V. Parameter optimization: force (F), time (T) and current intensity (I), in the RSW welding process of DP-290 steel plates using the Taguchi method. Soldagem e Inspeção. 2018;23(2):157-167. http://dx.doi.org/10.1590/0104-9224/si2302.04.

[19] Kumar D, Kumar J. Optimization of parameters in friction stir welding of AA6101-T6 by Taguchi. Singapore: Springer; 2020. http://dx.doi.org/10.1007/978-981-13-8196-6_32.

[20] Kumar S, Singh PK, Patel D, Prasad SB. Experimental investigation and optimization of welding parameters on TIG welding of stainless steel AISI 304 plates. Singapore: Springer; 2019. http://dx.doi.org/10.1007/978-981-13-2944-9_10.

[21] Hernández AB, De la Paz Guillon M, García LA. La metodología de Taguchi en el control estadístico de la calidad. Revista de La Escuela de Perfeccionamiento en Investigación Operativa. 2015;23(37):65-83.

[22] Indura. Manual de sistemas y materiales de soldadura. Santiago; 2002. 131 p.

[23] Lincoln Electric. Soldeo con alambre tubular. Toledo, España: Departamento de Formación, Lincoln-KD, S.A. p. 1-16.

[24] Universidad Tecnológica de Pereida. Soldadura: defectología y simbología. Colombia; 2015.

[25] Montgomery DC. Diseño y análisis de experimentos. 2. ed. Arizona: Limusa Wiley; 2004. 